Supporting Information

\title{
Fragile-Tough Mechanical Reversion of Silk Materials via Tuning Supramolecular Assembly
}

Xiaoyi Zhang ${ }^{\mathrm{a}}$, Liying Xiao ${ }^{\mathrm{a}}$, Zhaozhao Ding ${ }^{\mathrm{a}}$, Qiang $\mathrm{Lu}^{\mathrm{a}}{ }^{*}$, David L Kaplan ${ }^{\mathrm{b}}$

${ }^{a}$ National Engineering Laboratory for Modern Silk \& Collaborative Innovation Center of Suzhou Nano

Science and Technology, Soochow University, Suzhou 215123, China

bepartment of Biomedical Engineering, Tufts University, Medford, Massachusetts 02155, United

States

Corresponding author:

Qiang Lu, Tel: (+86)-512-67061649; E-mail: Lvqiang78@suda.edu.cn 

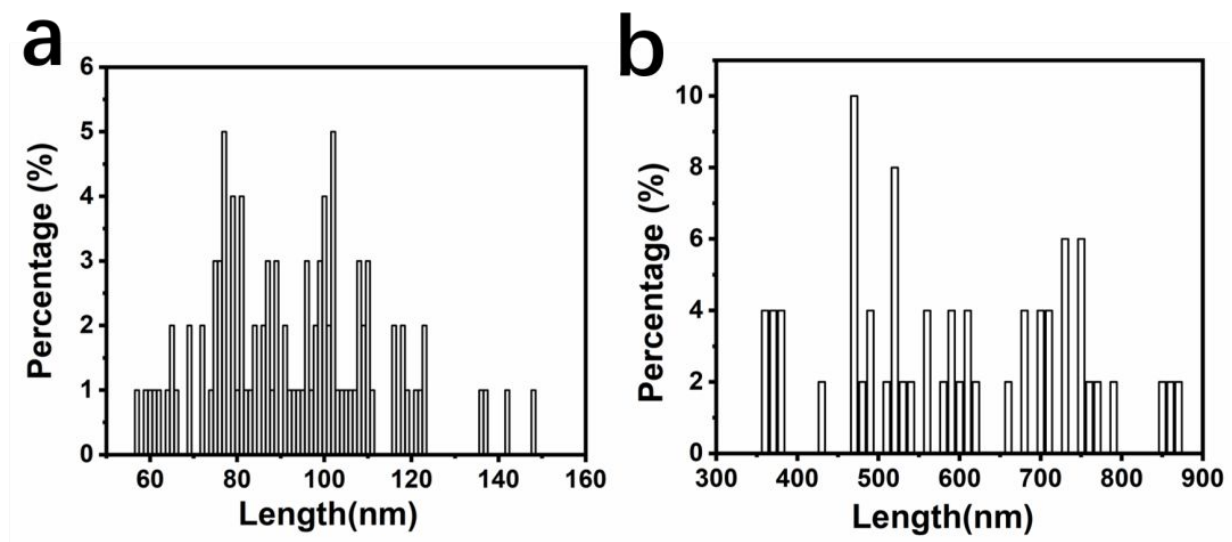

Figure S1. Length distribution of SNF when dispersed in different solvents (a) $\mathrm{SNF}_{2} \mathrm{H}_{2} \mathrm{O}$, (b) SNF-FA calculated from AFM images in Figure 1a, b.
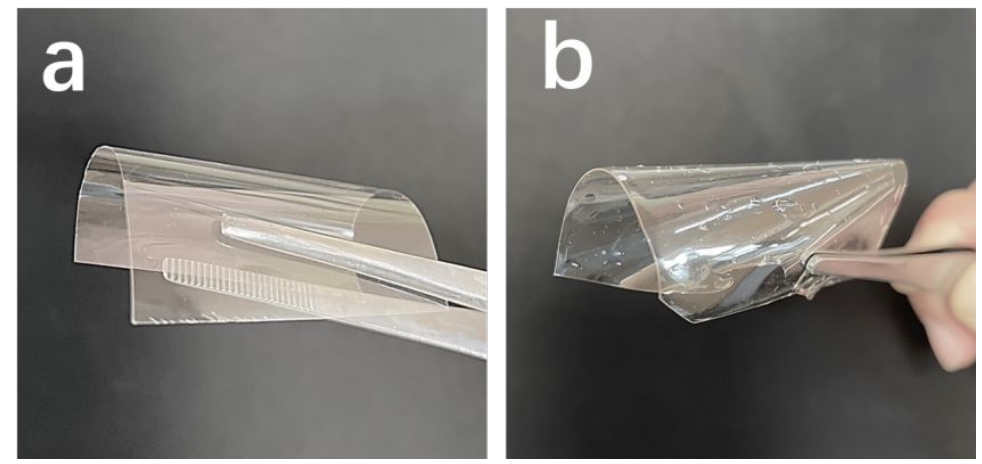

Figure S2. Photographs of SNF-FA film in bending state under dry and wet conditions.
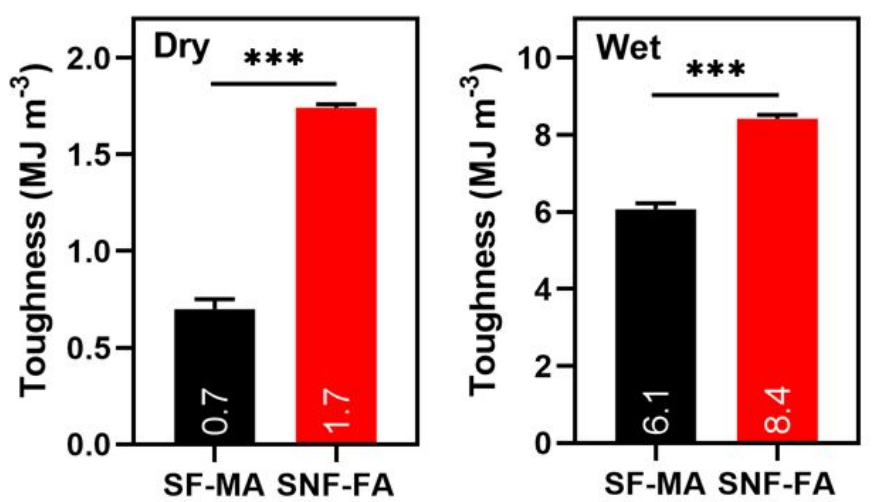

Figure S3. Toughness of silk films (SF-MA, SNF-FA) in the dry and wet state. 


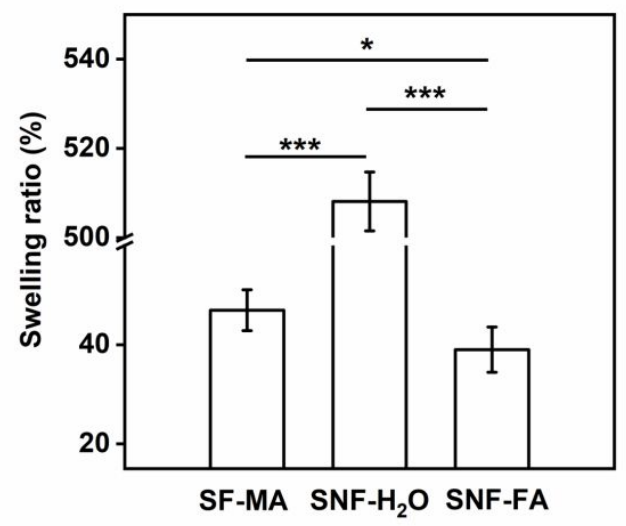

Figure S4. Swelling ratio of SF-MA, SNF- $\mathrm{H}_{2} \mathrm{O}$, and SNF-FA films after being immersed in distilled water at $37^{\circ} \mathrm{C}$ for $24 \mathrm{~h} . * \mathrm{P} \leq 0.05, * * \mathrm{P} \leq 0.01$, and $* * * \mathrm{P} \leq 0.001$ are considered statistically significant.

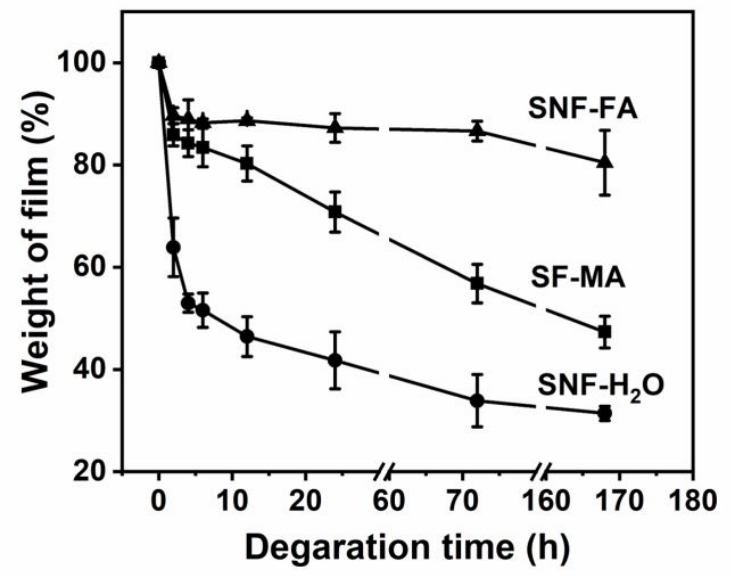

Figure S5. Enzyme degradation behavior of SF-MA, SNF- $\mathrm{H}_{2} \mathrm{O}$ and SNF-FA films incubated in protease XIV solution (5 $\mathrm{U} \mathrm{mL}^{-1}$ in PBS). 

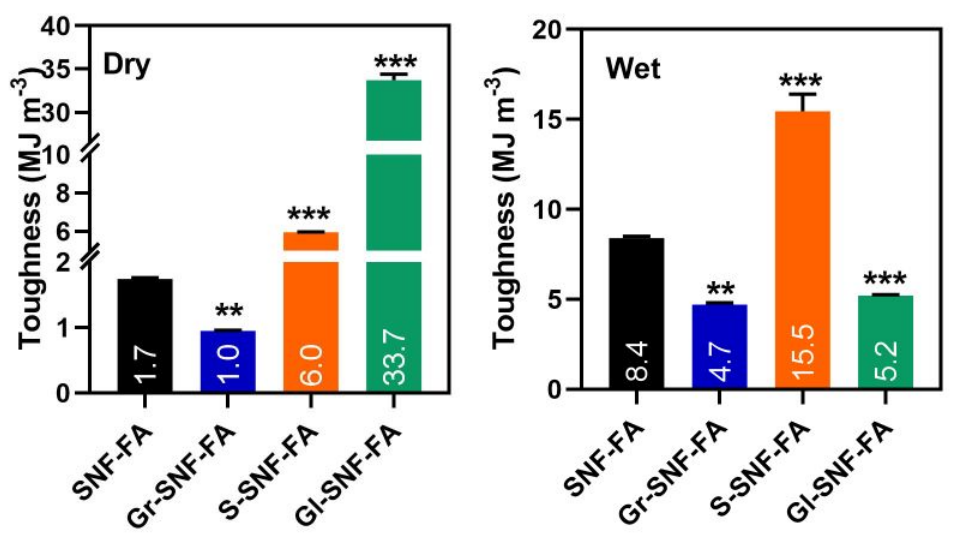

Figure S6. Toughness of different silk films in the dry and wet state. 